\title{
Multisensory integration and ADHD-like traits: Evidence for an abnormal temporal integration window in ADHD
}

\author{
Maria Panagiotidi*, Paul G. Overton and Tom Stafford \\ Department of Psychology, University of Sheffield, Western Bank, Sheffield, S10 \\ $2 \mathrm{TN}, \mathrm{UK}$ \\ * Present address: \\ School of Psychology, Sport and Exercise, \\ Staffordshire University, \\ College Road, \\ Stoke-on-Trent, \\ Staffordshire, ST4 2DE,
} UK

Address for correspondence:

Paul G. Overton,

Department of Psychology,

University of Sheffield,

Sheffield,

United Kingdom, S10 2TN.

Tel: $\quad+44(0) 1442226624$

Fax: +44 (0)1442766515

p.g.overton@sheffield.ac.uk 


\section{Abstract}

Abnormalities in multimodal processing have been found in many developmental disorders such as autism and dyslexia. However, surprisingly little empirical work has been conducted to test the integrity of multisensory integration in Attention Deficit Hyperactivity Disorder (ADHD). The main aim of the present study was to examine links between symptoms of ADHD (as measured using a self-report scale in a healthy adult population) and the temporal aspects of multisensory processing. More specifically, a Simultaneity Judgment (SJ) and a Temporal Order Judgment (TOJ) task were used in participants with low and high levels of ADHD-like traits to measure the temporal integration window and Just-Noticeable Difference (JND) (respectively) between the timing of an auditory beep and a visual pattern presented over a broad range of stimulus onset asynchronies. The Point of Subjective Similarity (PSS) was also measured in both cases. In the SJ task, participants with high levels of ADHD-like traits considered significantly fewer stimuli to be simultaneous than participants with high levels of ADHD-like traits, and the former were found to have significantly smaller temporal windows of integration (although no difference was found in the PSS in the SJ or TOJ tasks, or the JND in the latter). This is the first study to identify an abnormal temporal integration window in individuals with ADHD-like traits. Perceived temporal misalignment of two or more modalities can lead to distractibility (e.g., when the stimulus components from different modalities occur separated by too large of a temporal gap). Hence, an abnormality in the perception of simultaneity could lead to the increased distractibility seen in ADHD.

Keywords: multimodal integration, superior colliculus, simultaneity judgment, temporal order judgment 


\section{Introduction}

The ability to use multisensory integration (MSI) (i.e., integrate information from multiple sensory modalities) allows us to interact adaptively and efficiently with our surroundings by creating a unified and coherent internal representation of the external environment. For example, locating a predator depends on accurately detecting and integrating information from multiple sources (e.g. hearing the sound of the predator, detecting movement), while differentiating it from other stimuli (e.g. sound of the wind). Therefore, combining information across senses can significantly increase survival chances. Additionally, by effectively integrating stimuli from multiple modalities we avoid being overwhelmed by the constant input of information and we can attend to specific aspects of the environment. Multisensory integration allows us to accurately discriminate and/or detect unisensory stimuli (Schroeder \& Foxe, 2005; Shams, Wozny, Kim, \& Seitz, 2011).

Previous studies have shown that the temporal window of integration (TWI; i.e., how close together in time stimuli must occur in order to be perceptually integrated into a single, multisensory object) is highly variable across individuals (Stevenson, Zemtsov, \& Wallace, 2012) and abnormalities in multisensory integration have been observed in various disorders. For example, an extended TWI has been reported in dyslexia (Laasonen, Tomma-Halme, Lahti-Nuuttila, Service, \& Virsu, 2000; Hairston, Burdette, Flowers, Wood, \& Wallace, 2005). In addition, certain activities, such as musical training and video-game experience, have been associated with altered multisensory integration profiles (Petrini et al., 2009; Donohue, Woldorff, \& Mitroff, 2010)

Both empirical evidence and anecdotal accounts suggest the presence of sensory processing abnormalities in Attention Deficit Hyperactivity Disorder (ADHD), the 
most common neurodevelopmental disorder (Barkley 1997; Faraone, Sergeant, Gillberg, \& Biederman, 2003). Yochman and colleagues (2004) used the Sensory Profile questionnaire to examine sensory processing difficulties in children with ADHD and controls. Based on their mothers' perceptions, children with ADHD were reported to differ significantly from children without ADHD, one of the areas most affected being multisensory processing. Specifically, temporal processing, which is related to integrating information from multiple modalities, seems to be abnormal in children and adults with ADHD (Toplak, Dockstader, \& Tannock, 2006). In particular, a perceptual deficit in time discrimination affecting brief durations in the range of milliseconds has been observed (Smith, Taylor, Warner Rogers, Newman, \& Rubia, 2002; Marusich \& Gilden, 2014). Such deficits have also been found in non-clinical populations exhibiting ADHD-like traits (e.g., impulsivity) (Wittmann, Simmons, Flagan, Lane, Wackermann, \& Paulus, 2011; Baumann \& Odum, 2012). Sensitivity to such time intervals is essential to successfully integrating multimodal stimuli. However, despite the existing reports that point towards multisensory abnormalities in ADHD, surprisingly little empirical work has been conducted to actually test this.

The issue of potential multisensory integration abnormalities in ADHD is particularly pertinent at the present time given that a growing body of evidence suggests that the midbrain superior colliculus (SC) - a sensory structure which is intimately linked to orienting the eyes and head towards salient stimuli (Dean, Redgrave, \& Westby, 1989) - is dysfunctional in ADHD (reviewed by Overton, 2008; Overton and Clements, 2009). The SC is thought to play an important role in MSI. Visual, auditory, and somatosensory inputs converge onto a common pool of SC neurons, creating a substantial population of multisensory neurons (Meredith \& Stein, 1986). Neurons in the SC that receive input from multiple sensory modalities typically show enhanced 
responses to multisensory stimuli (compared to the largest unisensory response), provided that the stimuli from the two modalities are close together in space and time (Stein, Huneycutt, \& Meredith, 1988; Stein \& Meredith, 1993). Typically, multisensory stimuli will be temporally linked together if they occur within about $150 \mathrm{~ms}$ of each other (Stone et al., 2001; Powers, Hillock, \& Wallace, 2009; Donohue et al., 2010). The opposite effect (response depression) is observed when inputs are separated in space and time (Calvert, 2001; Calvert \& Thesen, 2004).

Due to its small size and its location, the human SC has only been examined directly in a small number of studies. However, a few human neuroimaging studies have reported SC activations in MSI paradigms (Calvert, 2001; Bushara, Grafman, \& Hallett, 2001; Miller, \& D'Esposito, 2005; Stevenson, Altieri, Kim, Pisoni, \& James, 2010; Powers, Hevey, \& Wallace, 2012). The SC seems to be part of a larger network involved in MSI, which includes the posterior parietal, superior temporal, prefrontal and insular cortices in addition to early visual and auditory areas and the posterior thalamus (Bushara et al., 2001, Calvert, 2001; Bushara, Hanakawa, Immisch, Toma, Kansaku, \& Hallett, 2003; Noesselt et al., 2007; Powers et al., 2012). The SC receives both ascending and descending input from visual, auditory, and somatosensory areas. Bushara and colleagues (2001) investigated the neural correlates of temporal synchrony detection between multimodal sensory inputs during an audiovisual simultaneity paradigm using PET. The SC showed significant functional interaction with the right insula - the region with the highest activity and the highest level of task-specific activity. Similar results were also reported in an fMRI study of audiovisual temporal correspondence by Calvert, Hansen, Iversen, and Brammer (2001). The paradigm they employed consisted of a visual stimulus (an $8 \mathrm{~Hz}$ reversing black-and-white checkerboard) and an auditory stimulus - $1000 \mathrm{~ms}$ white noise bursts - which were 
timed either to coincide precisely with the reversal rate of the visual checkerboard (matched experiment) or were randomly shifted out of synchrony (mismatched experiment). Calvert and colleagues (2001) found that the structure exhibiting the most significant cross modal facilitation and suppression to synchronous and asynchronous bimodal inputs respectively was the SC.

Evidence showing the involvement of the SC in MSI in humans also comes from behavioural studies. Frassinetti, Bolognini, and Làdavas (2002) and Bolognini, Frassinetti, Serino, and Làdavas (2005) investigated whether the spatial and temporal rules for MSI that have been previously observed in the animal SC (Stein \& Meredith, 1993) can be also found in humans. They used a unimodal visual and an across-modal audio-visual paradigm and showed that when an auditory stimulus was presented at one spatial location, it facilitated responses to a visual target at that location. The detectability of the visual stimuli, however, did not improve when the same visual and auditory stimuli were presented at spatially disparate loci. Furthermore, they found that the capacity of an auditory stimulus to enhance the detectability of a visual stimulus was evident only when the two stimuli were presented simultaneously. Their results suggest that human multisensory integration follows similar spatial and temporal rules to those that govern MSI at the collicular level (see also Odgaard, Arieh, \& Marks, 2003; Lovelace, Stein, \& Wallace, 2003; Noesselt et al., 2007), although the identity of the colliculus as the substrate underlying those rules in Frassinetti et al. (2002) and Bolognini et al. (2005) was inferred rather than determined.

The purpose of the present study was to examine links between symptoms of ADHD and the temporal aspects of multisensory processing, more specifically, the TWI - the period of time over which multisensory inter-actions are highly likely to occur. Hence, we examined multisensory processing in a healthy adult population with 
varying levels of ADHD-like traits. Given the role of the SC in multisensory processing and the proposed role of the SC in ADHD (Overton, 2008; Overton and Clements, 2009), the study provides an indirect test of the SC hypothesis. 


\section{Methods}

\section{Participants}

Forty seven participants (32 female) were recruited from the volunteers' list at a university in the north of England. The ages of the participants varied from 19 to 53 ( $M$ $=27.86, S D=7.29)$. All subjects had normal or corrected-to-normal vision and hearing and were naive as to the purpose of the experiment. None of the subjects reported having any history of neurological or psychiatric disorders or ADHD. Five participants were left-handed. The subjects all gave their informed consent to take part in the experiment and the procedures were carried out in accordance with the ethical standards of the Departmental Ethics Sub-Committee and British Psychological Society Guidelines. All participants were reimbursed for their time.

\section{Materials}

Although the participants were healthy and none were previously diagnosed with ADHD, ADHD psychopathology can be viewed dimensionally, with inattentive and hyperactive-impulsive symptoms distributed continuously in the general population (Hudziak et al., 2007; Rodriguez et al., 2007). Evidence at the level of molecular genetics also provides support for the hypothesis that ADHD represents the extreme end of traits present in the general population (Levy, Hay, McStephen, Wood, \& Waldman, 1997; Larsson, Anckarsater, Råstam, Chang, \& Lichtenstein, 2012; Martin, Hamshere, Stergiakouli, O'Donovan, \& Thapar, 2014). The approach of using the general population has been very popular in studies on other developmental disorders such as autism spectrum disorder (ASD) (Dickinson et al., 2014) and has been recently employed by researchers investigating ADHD (Biehl, Ehlis, Müller, Niklaus, Pauli, \& Herrmann, 2013; Polner, Aichert, Macare, Costa, \& Ettinger, 2015). Hence, in the 
present study, we used healthy adult participants with a range of ADHD-like traits, as measured by the World Health Organization Adult ADHD Self-Report Scale (ASRS; Kessler et al., 2005).

The ASRS is a questionnaire which was developed in conjunction with the revision of the WHO Composite International Diagnostic Interview (CIDI) (Kessler \& Üstün, 2004). The ASRS contains eighteen items from the DSM-IV-TR (American Psychiatric Association, 2000) and measures the frequencies of symptoms. Subjects are asked to report how often they experience each symptom in a period of six months on a five-point Likert scale which ranges from 0 for never, 1 for rarely, 2 for sometimes, 3 for often, and 4 for very often (Kessler et al., 2005). The ASRS has a two factor structure (Reuter, Kirsch, \& Hennig, 2006), which includes an inattention subscale and a hyperactivity/impulsivity subscale, each containing nine items. The reliabilities (Cronbach's alpha) for the two subscales of inattention (.75) and impulsivity (.77) as well as for the total ASRS (.82) are satisfactory (Reuter et al., 2006). The original questionnaires are formatted with darkly shaded boxes for certain items which signify more severe symptoms. We removed the darkly shaded boxes in the ASRS to minimize any possibility that the darker shaded areas may motivate symptom exaggeration by the participants.

Two tasks were administered to examine MSI; a Simultaneity Judgement task (SJ) and a Temporal Order Judgement task (TOJ). Simultaneity judgement and TOJ tasks are thought to tap into somewhat different underlying mechanisms (van Eijk, Kohlrausch, Juola, \& van de Par, 2008; see Discussion). Each task is described in detail below. 


\section{Simultaneity Judgement Task}

A Simultaneity Judgement task was employed similar to the one described by Donohue and colleagues $(2010,2012)$. Multisensory stimuli (an auditory beep and a visual pattern) were presented over a broad range of stimulus onset asynchronies (SOAs) using OpenSesame (Mathôt, Schreij, \& Theeuwes, 2012) with a PsychoPy (Peirce, 2007) back-end. The stimuli used for this task were a black and white square checkerboard (5 x 5) and an auditory tone (60dBSL, $5 \mathrm{~ms}$ rise-and-fall time, $1200 \mathrm{~Hz}$ ), presented centrally. Both stimuli were presented for $33 \mathrm{~ms}$. Subjects were seated 50-60 $\mathrm{cm}$ in front of a 20 inch screen (Mitsubishi Diamondpro 2070sb; refresh rate $86 \mathrm{~Hz}$ ). The auditory and visual stimuli were presented at thirteen stimulus onset asynchronies (SOAs in ms: $-300,-250,-200,-150,-100,-50,0,50,100,150,200,250,300$ ), where negative SOAs represent the auditory stimulus appearing first and positive SOAs represent the visual stimulus appearing first, and 0 represents physical simultaneity. The typical range for the window of integration is -150 to $150 \mathrm{~ms}$. As a result, approximately half of the SOAs presented might be expected to be perceived as simultaneous even though only one is objectively simultaneous. The participants were instructed to press different keys to indicate whether the stimuli were presented simultaneously or asynchronously. Participants were asked to determine if the auditory and visual stimuli occur at the same time or at different times by making self-paced key-press responses; one key (' $\mathrm{S}$ ') was associated with presentations that were perceived as "simultaneous" and another (' $\mathrm{J}$ ') with those perceived as "not simultaneous".

\section{Temporal-Order Judgement Task}

Temporal Order Judgement (TOJ) tasks are similar to SJ tasks. Observers are presented 
with two stimuli (auditory and visual in our case) at a range of temporal offsets and are asked to choose which of the two appeared first. The same stimuli were used for the TOJ task as in SJ task. Twelve SOAs were presented to the participants $(-300,-250,-$ $200,-150,-100,-50,50,100,150,200,250,300)$. Participants were asked to judge whether the auditory or the visual stimulus was presented first, again indicated with a keypress ('c', auditory first; 'm', visual first). The participants were instructed to be as accurate as possible, and that there was not a response time limit.

\section{Procedure}

Before signing up, participants were screened using an online version of the ASRS, which was presented as a personality test. Participants with ASRS scores over 35 were assigned to the group with high levels of ADHD-like traits (referred to as the 'Hi ADHD group' for brevity). The cutoff score was based on the results of a previous scoping study by the authors examining ADHD-like traits in a sample of 800 volunteers from the general population based in a city in the north of England (Panagiotidi, Overton, \& Stafford, unpublished). In this relatively large sample, the mean ASRS score was 31.83, and a score of 35 (the cutoff in the current study) was at the 75th percentile. Previous studies have reported similar cutoff points (e.g. 34 according to Stark and colleagues, 2011). In the present study, participants with ASRS scores under 29 were assigned to the 'Lo ADHD group'. If the participant's score fell within 29 and 35 they were not allowed to take part in the study. The experimenter was blind as to which group the participant belonged at the time of testing.

The complete experimental session lasted approximately $45 \mathrm{~min}$. All participants were first presented with the SJ task and were requested to complete a practice block of 13 trials. The practice session was followed by 260 test trials ( 20 trials 
for each of the 13 SOAs). The SOAs were presented in a different random order for each participant. After finishing the SJ task, participants had a short break during which they filled in a demographics questionnaire. Once they were ready for the second part of the study, the TOJ task was presented. Initially, participants completed a practice block of 24 trials. The practice session was followed by 288 test trials ( 24 trials for each of the 12 SOAs). Again, the SOAs were presented in a different random order for each participant. The order of the task was kept the same for all participants, as it has been shown that SJs are susceptible to the effects of adaptation to temporal asynchronies (Vatakis, Navarra, Soto-Faraco, \& Spence, 2007).

\section{Data Analysis}

The primary measure of the SJ task was the proportion of trials reported as 'simultaneous' at each SOA. The data from each participant were fitted to a Gaussian function using a nonlinear least-squares fit (as in Zampini, Shore, \& Spence, 2003; Donohue et al., 2010, 2012), and the two sides of the Gaussian were allowed to vary independently. Based on the above fit, a point of subjective simultaneity (PSS) was calculated, which reveals the specific SOA at which participants are most likely perceiving the auditory and visual stimuli as occurring simultaneously. A negative SOA would mean that the observer has a bias to perceive auditory information before visual and a positive SOA would mean that the observer has a bias to perceive visual information before auditory. The TWI was defined as the width of each participant's distribution at half height.

For the TOJ task, the proportion of visual first responses was calculated for each participant for each SOA. The data from each participant were fitted with a Gaussian cumulative distribution function to further investigate the differences between the two 
groups (Donohue et al., 2012). The PSS was calculated from the $50 \%$ point of the TOJ curve. The $50 \%$ point on the function denotes the physical temporal offset at which the observer is maximally uncertain as to which of the two stimuli came first. The slope of the psychometric function can be used to measure sensitivity to temporal asynchrony, in the form of a Just-Noticeable Difference (JND)(Donohue et al., 2012). JustNoticeable Differences represent a numerical estimate of the sensitivity of the participant to changes in the stimulus characteristics. Specifically, a high sensitivity to asynchrony would allow the participant to notice small changes in the physical temporal offset between the two stimuli. High sensitivity to asynchrony would be expressed as a low JND and relatively steeply sloping psychometric function.

A mean score for the proportion of responses reported as simultaneous for audio-first (SOAs: -300, -250, -200, -150, -100, -50) and visual-first (50, 100, 150, 200, $250,300)$ responses was calculated for each participant. In addition to this, differences between groups in the probability of simultaneity report at each SOA were examined. 


\section{Results}

ASRS Scores

Scores on the ASRS checklist varied from 7 to 66 , with a mean score of $33.28(S D=$ 13.03). The mean score on the inattention subscale was $17.36(S D=6.54)$ and the hyperactivity subscale $15.96(S D=7.57)$. The two subscales were correlated, $r(47)=$ $.698, p<.01$ (2-tailed) and the overall ADHD score was correlated with both the inattention $(r(47)=.909, p<.01)$ and the hyperactivity subscale $(r(47)=.933, p<.01)$. Since the two subscales were strongly correlated with each other and the overall ASRS scores, only overall ASRS scores were used in our analyses.

The participants were categorised into a two groups based on their overall ASRS scores. Participants with scores under 29 were assigned to the Lo ADHD group and participants with scores above 35 (close to the mean ASRS score) were assigned to the Hi ADHD group. The latter group contained individuals with scores in excess of 47, scores considered by Stark et al. (2011) to indicate that the subject was "most likely to have ADHD". Initially, equal numbers of high and low ADHD participants were recruited but the dropout rate was greater in the Hi ADHD group, resulting in final numbers of 26 (22 female) participants in the Lo ADHD group and 21 (10 female) in the Hi ADHD group.

\section{Simultaneity Judgement Task}

Ten participants were disqualified from the analysis as they had PSS and/or TWI values over $600 \mathrm{~ms}$ (i.e., they fell outside the SOA range tested; cf. Spence, Shore, \& Klein, 2001, Vatakis et al., 2007 for similar exclusion criteria), indicating that these participants could not perform the task. Such exclusion rates are common in studies examining the temporal aspects of MSI. As a result, the eventual Hi ADHD group for 
the SJ analysis comprised 17 participants (10 female) and the Lo ADHD group 20 participants (17 female). Independent samples t-tests were conducted to test for differences in the performance of the Lo and Hi ADHD groups. There was a significant difference in the width of the TWI in the Lo $(M=396.1, S D=89.34)$ and the Hi $(M=$ $326.6, S D=93.04)$ ADHD group $(t(35)=2.33, p=.026)$, with a moderate to large effect size $(d=.76)$. The Hi ADHD group had a significantly smaller window of integration compared to the Lo ADHD group (Figure 1). There was a small (but nonsignificant) correlation between ASRS scores across the groups and the TWI $(r(37)=$ $.28, p=.09)$. Although the left and right side of the Gaussian function were allowed to vary independently during the fitting process, no systematic between group differences in symmetry were apparent.

No difference was found between the Lo $(M=-20.21, S D=58.94)$ and Hi $(M$ $=-43.64, S D=36.74)$ ADHD groups in the PSS, $t(35)=1.42, p=.165, d=.48$, with both groups reporting a tendency for visual stimuli presented after auditory stimuli to be perceived as simultaneous (although across the groups, the correlation between ASRS scores and the PSS approached significance; $r(37)=-.29, p=.07)$. However, there was a significant difference between the Lo and Hi ADHD groups in the proportion of visual-first, $t(35)=2.17, p=.036, d=.71$, and audio-first SOAs, $t(35)=$ 2.07, $p=.046, d=.68$, declared to be simultaneous, with the Lo ADHD group making a higher proportion of simultaneous responses in both cases (sound first, Lo: $M=0.63$, $S D=0.13 ; \mathrm{Hi}, M=0.53, S D=0.15$; visual first, Lo: $M=0.50, S D=0.23 ; \mathrm{Hi}, M=0.35$, $S D=0.21)$

Given that there was a higher proportion of females in the Hi ADHD group than the Lo, an independent samples t-test was conducted to identify potential effects of gender on performance in the task. No differences were found between males $(M=$ 
$327.5, S D=107.5)$ and females $(M=381.5, S D=87.65)$ in the width of the TWI $(t(35)$ $=-1.63, \mathrm{p}=.112, d=.55)$, or between males $(M=-30.99, S D=47.62)$ and females $(M$ $=-30.97, S D=53.11)$ in the PSS $(t(35)=-.001, p=.999, d=.40)$. Furthermore, although the proportion of males was greater in the Hi ADHD group than the Lo, additional males in the Hi ADHD group cannot account for the significant difference between the TWI in the Hi and Lo ADHD groups. The width of the TWI was smaller in the Hi ADHD group, whereas males had somewhat larger TWIs than females in the Lo ADHD group $(M=454.0667, S D=23.31$ vs $M=385.87, S D=93.11 ; t(18)=1.23$, $\mathrm{p}=.232, d=1.00)$ 


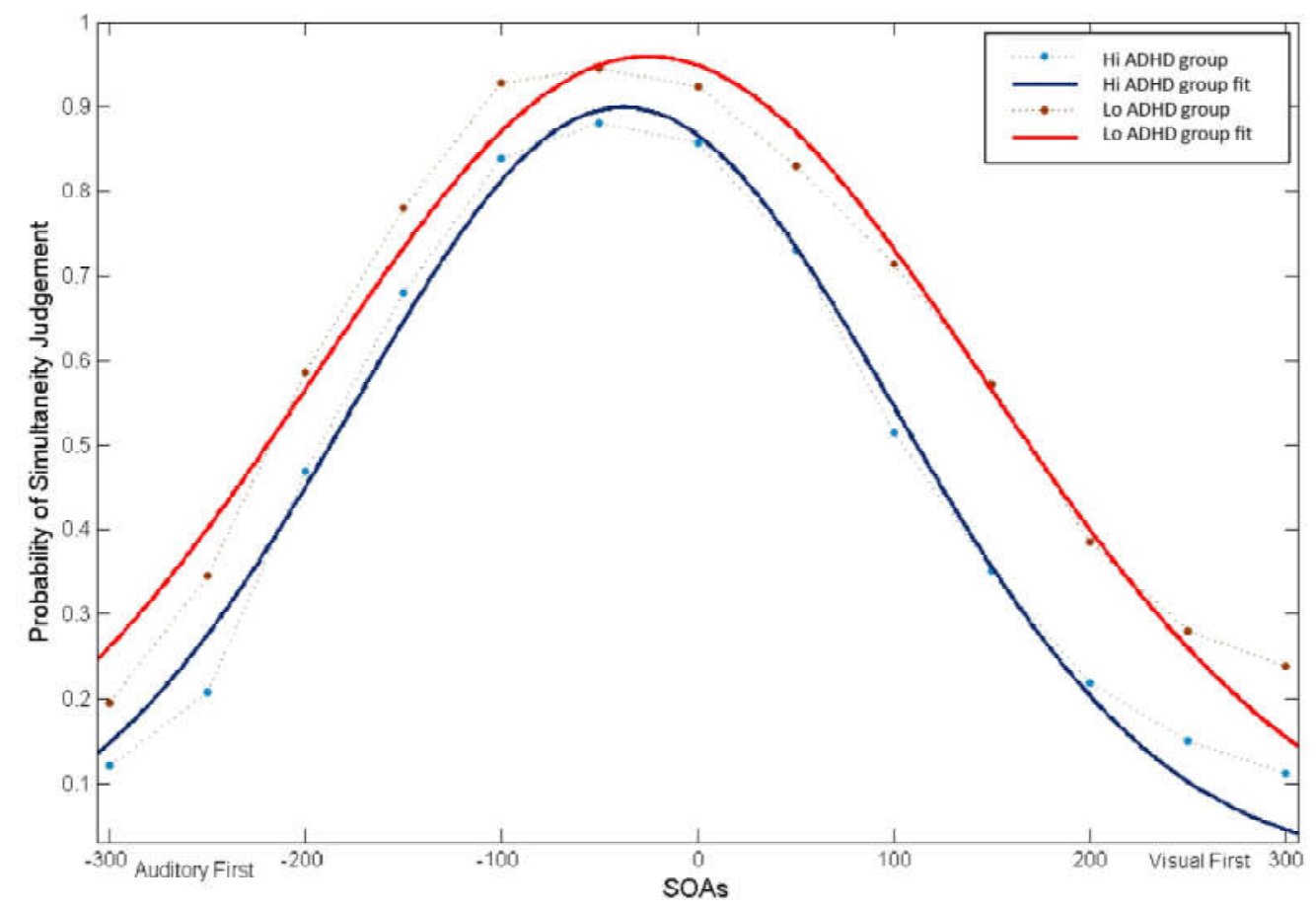

Figure 1: Simultaneity Judgement Task performance: Mean proportion of simultaneous responses (and lines of best fit through the responses) as a function of the stimulus onset asynchrony between the auditory and the visual stimulus in Hi and Lo ADHD groups. The curve's peak gives the point of subjective simultaneity (PSS) and the temporal window of integration (TWI) is defined as the width of the distribution at half height. The PSS did not differ between groups but the Hi ADHD group had narrower TWIs. SOAs $=$ Stimulus Onset Asynchronies.

\section{Temporal Order Judgement Task}

Data from 10 participants were excluded from the TOJ task analysis due to poor performance (i.e., accuracy under 50\% in the TOJ task, which resulted in their data not being fitted). As a result, the eventual Hi ADHD group for the TOJ analysis comprised 17 participants (10 female) and the Lo ADHD 20 participants (17 female). There was no significant difference in the JND between the Lo $(M=169.67, S D=70.35)$ and the 
$\operatorname{Hi}(M=148.11, S D=43.66) \operatorname{ADHD}$ group $(t(35)=1.076, p=.289, d=.37$; Figure 2$)$. Likewise, no difference was found between the Lo $(M=5.82, S D=105.33)$ and $\mathrm{Hi}(M$ $=27.77, S D=81.98)$ ADHD groups in the PSS, $t(35)=-.689, p=.495, d=.23$, with both groups reporting a tendency for auditory stimuli presented after visual stimuli to be perceived as simultaneous. Accuracy scores were slightly higher in the ADHD group (Table 1), although this difference was not significant $(t(35)=-1.888, p=.068, d=$ .60). Again, across the groups, correlations between ASRS scores and the JND, and PSS, were non-significant $(r(37)=-.13, p=.43$, and $r(37)=.18, p=.29$, respectively).

Given the apparent difference in performance of the Hi and Lo ADHD groups in the SJ and TOJ tasks, that comparison was statistically assessed using a two-way ANOVA (factors Group [Hi ADHD, Lo ADHD] and Task [SJ and TOJ]). Analysis revealed a statistically significant main effect of Group, $F(2,33)=5.64, p<.05, \eta^{2}=.14$., and Task, $F(3,33)=10.1, p<.01, \eta^{2}=.48$. However, the interaction between Group and Task was not significant $(F(3,33)=1.37, p=.26, \eta 2=.11)$. 


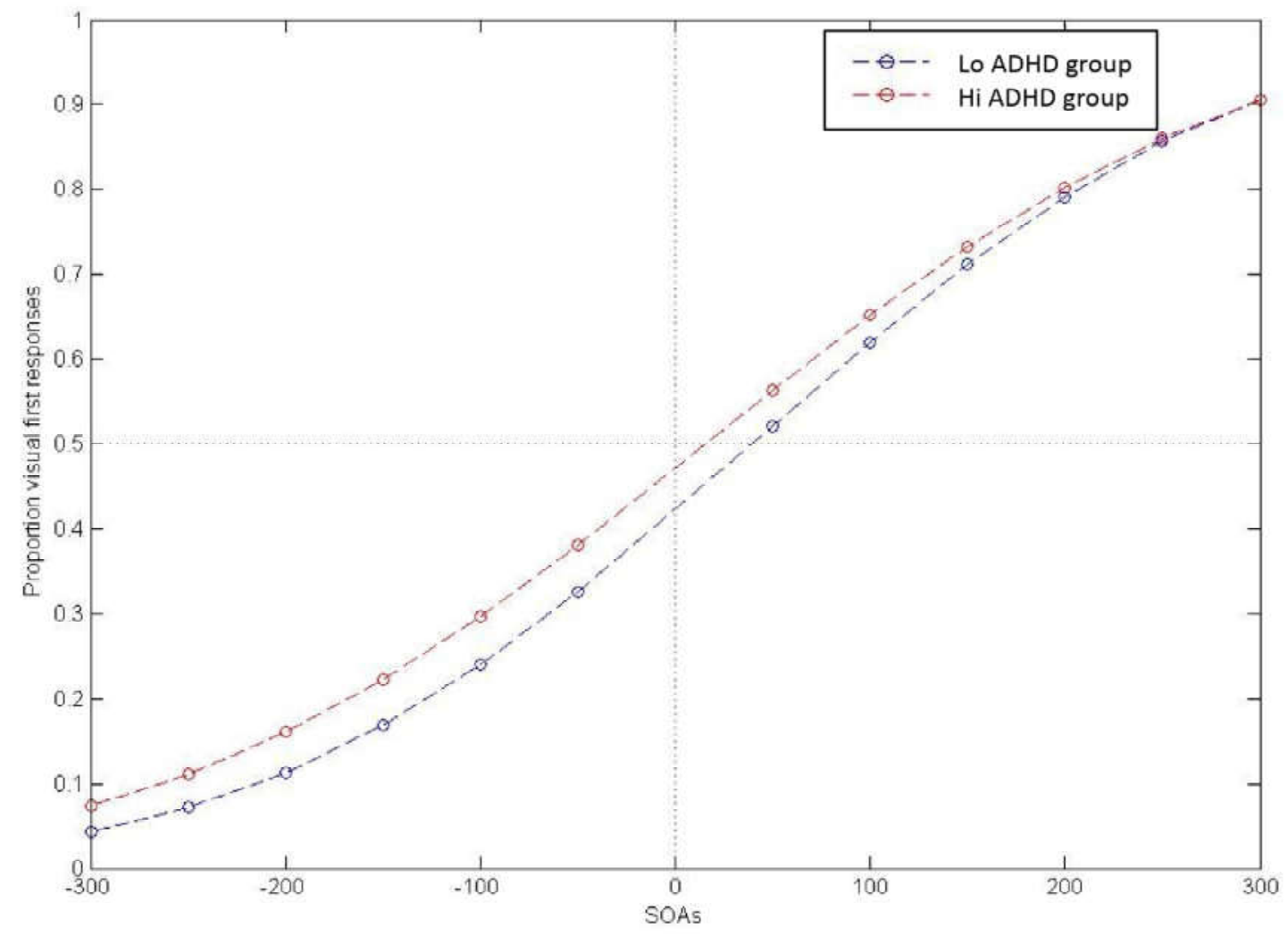

Figure 2: Temporal Order Judgement Task performance: Mean proportion of visual first responses as a function of the stimulus onset asynchrony between the auditory and the visual stimulus in Hi and Lo ADHD groups. The Point of Subjective Simultaneity (PSS) is defined as the 50\% point on the curve, and the slope of the curve gives a measure of sensitivity to temporal asynchrony (Just-Noticeable Difference; JND). Neither the PSS nor the JND differed significantly between the groups. SOAs = Stimulus Onset Asynchronies. 
Table 1: Accuracy in the Temporal Order Judgement task in the Lo and Hi ADHD groups

\begin{tabular}{lcrr}
\hline Variable & $\boldsymbol{n}$ & $\boldsymbol{M}$ & $\boldsymbol{S D}$ \\
\hline Accuracy in TOJ & & & \\
Lo ADHD & 21 & 78.89 & 10.42 \\
Hi ADHD & 16 & 83.95 & 5.66 \\
Visual first & & & \\
Lo ADHD & 21 & 81.30 & 14.41 \\
Hi ADHD & 16 & 85.61 & 9.83 \\
Sound first & & & \\
Lo ADHD & 21 & 76.49 & 14.3 \\
Hi ADHD & 16 & 82.29 & 7.28 \\
\hline \multicolumn{3}{l}{ M mean, SD = Standard Deviation }
\end{tabular}




\section{Discussion}

When tested on an SJ task, participants with high levels of ADHD-like traits were found to have a significantly smaller TWI than participants with low levels of ADHD-like traits. No significant difference was found in the PSS between the groups. The Hi ADHD group judged more SOAs as not being simultaneous both in visual-first and audio-first trials. Insofar as the TWI is related to the ability to create unified multisensory perception, individuals with narrower windows are more likely to dissociate temporally asynchronous inputs. Previous studies have found that the width of the TWI, specifically the right side of an individual's TWI, where the auditory stimulus follows the visual, is significantly correlated with the strength of illusory percepts (Stevenson et al., 2012). Perceived temporal misalignment of two or more modalities can lead to distractibility (e.g., when the stimulus components from different modalities occur separated by too large of a temporal gap; Figure 3). An abnormality in the perception of simultaneity could increase distractibility, which is a core symptom of ADHD (e.g. Thorley, 1984).

In contrast to performance on the SJ task, no significant differences were found between Hi and Lo ADHD groups on the TOJ task. The PSS and the JND were similar in both groups (although some caution is required here and below because of the somewhat small sample size). When examining accuracy of responses across SOAs, a trend was observed; participants with high levels of ADHD-like traits tended to make more accurate estimations (i.e, they were more likely to accurately judge the order in which the tone and the checkerboard were presented). However, again, this difference between the groups was not significant.

To our knowledge, the present study is the first to report abnormalities in MSI in individuals with high levels of ADHD-like traits. Even though sensory issues are 
often reported in patients with ADHD, this area of research has been comparatively neglected. However, the difference between the Hi and Lo ADHD groups was found to be significant only in the SJ task (although the significant main effect of Group but lack of interaction in our ANOVA comparing the two tasks suggests that there may be a trend towards differential performance in the TOJ task as well). In the previous literature, TOJ tasks and SJ tasks appear to have been used almost interchangeably to measure perceived simultaneity (Vatakis, Navarra, Soto-Faraco, \& Spence, 2008). On this basis, one would expect to be able to identify differences between the groups on both tasks. That said, a well-established finding in the field is that SJs and TOJs are supported by different perceptual and brain mechanisms (Vatakis et al., 2007; van Eijk et al., 2008; Love, Petrini, Cheng, \& Pollick, 2013). More specifically, different aspects of temporal judgements are required for each task. The perception of successiveness is a necessary requirement for the perception of temporal order required in the TOJ (Allan \& Kristofferson, 1974). The PSS estimate obtained in a TOJ task is shifted in the direction of the most sensitive part of the synchrony judgement curve, which is obtained in the SJ task. This results in a response bias that may affect the PSS by affecting the mid-point of the psychometric function (Vatakis et al., 2008).

Support for this was provided by a study by van Eijk et al. (2008), whose results showed that TOJ PSS values showed no significant correlations with SJ values. Measures of observer sensitivity to asynchrony also showed no correlation between TOJ and SJ task. From their data, van Eijk and colleagues concluded that TOJ and SJ measure different aspects of observers' perceived simultaneity. Evidence suggests that different neural networks might be involved in SJ and TOJ tasks. Specifically, a cortical and subcortical network comprising the insula, cerebellum, inferior frontal gyrus, inferior parietal lobe, superior colliculus and posterior thalamus seems to be responsible 
for detection of asynchrony in a SJ task (Bushara et al., 2001). In contrast, Fink and colleague (Fink, Ulbrich, Churan, \& Wittmann, 2006) proposed that there might be more than three different neural mechanisms mediating TOJ. Furthermore, the TOJ task has been found to be more complex and require more resources (e.g., decision making) than the SJ task (Yarrow, Shapiro, DiCosta, \& Arnold, 2014). In general, the majority of published work suggests that the SJ task provides a more sensitive measure regarding the temporal aspects of a stimulus (i.e., the PSS measure; Schneider \& Bavelier, 2003; Vatakis et al., 2008).

The lack of significant differences in the TOJ task and the findings from the SJ task could pinpoint the perceptual and neural mechanisms involved in ADHD. The SC is one of the main areas involved in the SJ task (Bushara et al., 2001; Meredith, Nemitz, \& Stein, 1987). Abnormal SC function would most likely lead to an altered TWI. In particular, given that evidence suggests the SC is hyper-responsive to sensory stimuli in ADHD (Overton, 2008; Overton and Clements, 2009), better resolution of unimodal sensory events might be expected, leading to the observed decrease in the tendency to consider non-simultaneous stimuli as simultaneous (as indicated by smaller integration window in the high ADHD group). We propose that collicular hyper-sensitivity brings with it the 'advantage' of better signal to noise, and thus sensitivity to all stimuli is likely to be affected. Changing stimulus intensity has been shown to increase the likelihood that a non-simultaneous pair of visual and auditory stimuli will be perceived as such (Smith, 1933), and we believe that the increase in neural responsiveness in participants with high levels of ADHD-like traits is effectively the neural correlate of an increase in stimulus intensity. Although an abnormality in the perception of simultaneity might be expected to increase distractibility, recent evidence suggests that the temporal integration window can be manipulated (Fujisaki, Shimojo, Kashino, \& 
Nishida, 2004; Stevenson, Wilson, Powers, \& Wallace, 2013; Powers et al., 2009, 2012; Mégevand, Molholm, Nayak, \& Foxe, 2013). Thus, it might be possible to develop behavioural interventions to normalise the window of integration in ADHD. Enhanced perception in various tasks has been observed in other disorders such as ASD (visual acuity; Ashwin, Ashwin, Rhydderch, Howells, \& Baron-Cohen, 2009, visual search; O’Riordan, Plaisted, Driver, \& Baron-Cohen, 2001, attention to detail; Smith \& Milne, 2009) and dyslexia (creativity; Everatt, Steffert, \& Smythe, 1999). A smaller integration window could be seen as advantageous. Being able to parse audio-visual information when they occur closely together in time could reduce uncertainty in certain situations (Love, Pollick, \& Petrini, 2012). Intriguingly, although ASD is associated with enhanced perception in some domains, unlike ADHD, it is associated with a broadening on the TWI (e.g. Foss-Feig et al., 2010). The pathophysiology of ASD (like ADHD) is poorly understood, however the proposal has been made that under some circumstances, signal to noise could be decreased in ASD (Rubenstein, \& Merzenich, 2003). If that is the case, then reduced signal to noise will most likely lead to a broader TWI, in the same way as increased signal to noise in ADHD leads to a reduced TWI (see above).

A curious finding in our study is the negative PSS reported for the SJ task. This is contrary to the popular opinion that an auditory stimulus has to be presented after a visual stimulus to be perceived as simultaneous (as in the TOJ task; Hirsh \& Sherrick Jr, 1961; Dinnerstein \& Zlotogura, 1968; Zampini et al., 2003; Keetels \& Vroomen, 2005). Only a limited number of studies report the opposite in SJ tasks (van Eijk et al., 2008). A possible explanation for this according could be the higher intensity of the visual stimuli and/or lower intensities of the auditory stimuli. Stimulus intensity is a factor that seems to influence perceived simultaneity (Boenke, Deliano, \& Ohl, 2009). 
The visual stimulus we used was a high-contrast black and white checkerboard, while the auditory stimulus consisted of a simple, low volume, sinewave. Most studies employ flashes or simpler visual stimuli.

It is unclear whether the abnormal performance of the Hi ADHD group is associated with abnormal multimodal perception outside the lab. This is partly due to the low ecological validity of our paradigm. A number of ecologically valid MSI paradigms (the bouncing ball; Lewkowicz, 1996, audio and visual looming stimuli; Maier, Neuhoff, Logothetis, \& Ghazanfar, 2004) have been developed. In tasks such as the bouncing ball, a green disc is perceived as a moving stimulus, thus leading to an event with a causal interpretation. As a result, a more clear and predictable temporal relation exists (the visual component is expected to lead the auditory component). Future studies should attempt to replicate our results using such an ecologically valid paradigm.

In summary, integrating stimuli from multiple senses is a skill integral to survival which is affected in a range of developmental disorders, although so far, it has been neglected in ADHD research. We investigated possible relationships between ADHD-like traits and measures of MSI in a group of adults with high and low levels of ADHD-like traits as measured by a self-report questionnaire. Differences were found between participants with high and low ADHD in an SJ task (but not a TOJ task), where participants with high levels of ADHD-like traits were found to have a significantly smaller TWI than participants with low levels of ADHD-like traits. These findings hearald a new area of study for ADHD research and shed light to the possible mechanisms involved in the disorder. 


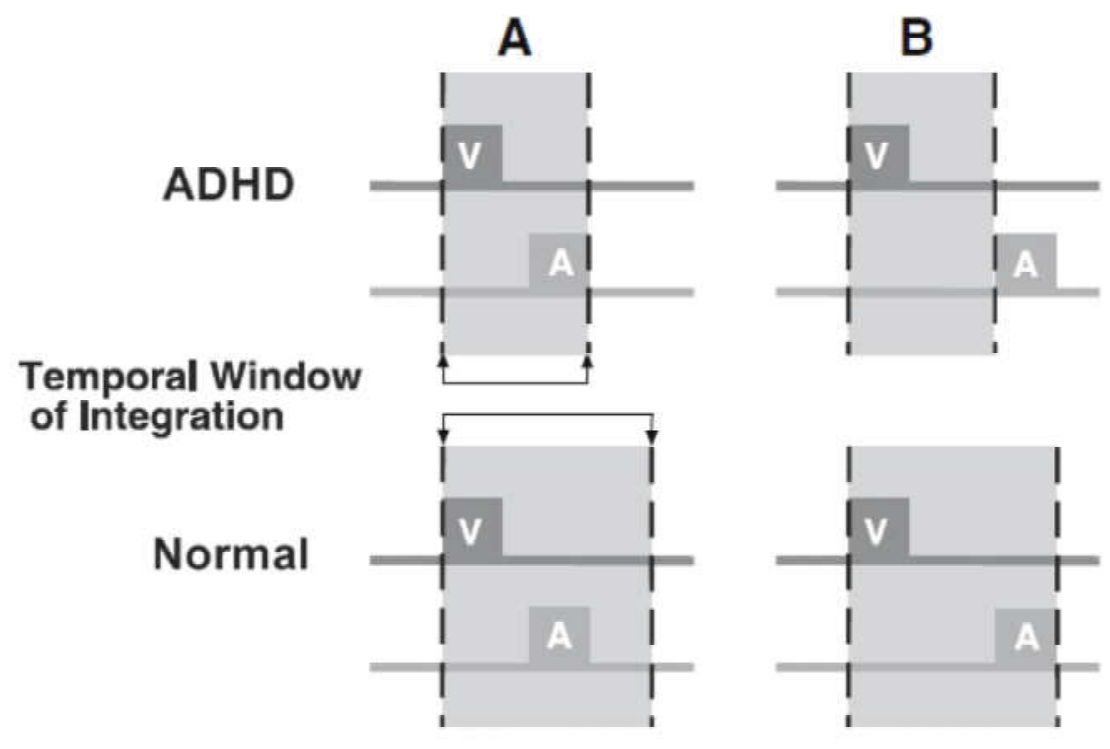

Figure 3: Model in which the temporal window of multisensory integration is narrowed in ADHD (a). Visual (V) and auditory (A) stimuli separated by small temporal intervals are processed as a unified, integrated event by both people with high ('normal') and low levels of ADHD-like traits ('ADHD'); (b). Individuals with high levels of ADHDlike traits experience two stimuli with larger temporal separations as distinct in situations where those with low levels of ADHD-like traits consider the stimuli to form an integrated, unified percept. 


\section{References}

American Psychiatric Association, Diagnostic and statistical manual of mental disorders: DSM-5, 5 ${ }^{\text {th }}$ Edition, Autor, Washington, DC, 2013.

Barkley, RA. (1997) Behavioral inhibition, sustained attention, and executive functions: Constructing a unifying theory of ADHD. Psychological Bulletin, 121 (1) $65-94$

Barry, TD., Klinger, LG., Lyman, R., Bush, D., Hawkins, L. (2001) Visual selective attention versus sustained attention in boys with attention-deficit/hyperactivity disorder. Journal of Attention Disorders, 4 (4) 193-202.

Baumann, AA. Odum, AL. (2012) Impulsivity, risk taking, and timing. Behavioural Processes, 90 (3) 408-414.

Biehl, SC., Ehlis, A-C., Müller, LD., Niklaus, A., Pauli, P., Herrmann, MJ. (2013). The impact of task relevance and degree of distraction on stimulus processing. BMC Neuroscience, 14 (1) 107.

Bolognini, N., Frassinetti, F., Serino, A., Làdavas, E. (2005) "Acoustical vision” of below threshold stimuli: interaction among spatially converging audiovisual inputs. Experimental Brain Research, 160 (3) 273-282.

Breier, JI.. Gray, LC., Fletcher, JM., Foorman, B., Klaas, P. (2002) Perception of speech and nonspeech stimuli by children with and without reading disability and attention deficit hyperactivity disorder. Journal of Experimental Child Psychology, 82 (3) $226-250$.

Breier, JI.. Gray, L., Fletcher, JM., Diehl, RL., Klaas, P., Foorman, BR., Molis, MR. (2001) Perception of voice and tone onset time continua in children with dyslexia with and without attention deficit/hyperactivity disorder. Journal of Experimental Child Psychology, 80 (3) 245-270. 
Brown, LN., Vickers, JN. (2004) Temporal judgments, hemispheric equivalence, and interhemispheric transfer in adolescents with attention deficit hyperactivity disorder. Experimental Brain Research, 154 (1) 76-84.

Bushara, KO., Grafman, J., Hallett, M. (2001) Neural correlates of auditory visual stimulus onset asynchrony detection. Journal of Neuroscience, 21 (1) 300-304.

Bushara, KO., Hanakawa, T., Immisch, I., Toma, K., Kansaku, K., Hallett, M. (2003) Neural correlates of cross-modal binding. Nature Neuroscience, 6 (2) 190-5.

Calvert, GA. (2001) Crossmodal processing in the human brain: insights from functional neuroimaging studies. Cerebral cortex, 11 (12) 1110-1123.

Calvert, GA., Hansen, PC., Iversen, SD., Brammer, MJ. (2001) Detection of audiovisual integration sites in humans by application of electrophysiological criteria to the bold effect. Neuroimage, 14 (2) 427-438.

Calvert, GA., Thesen, T. (2004) Multisensory integration: methodological approaches and emerging principles in the human brain. Journal of Physiology-Paris, 98 (1) $191-205$.

Foss-Feig, JH., Kwakye, LD., Cascio, CJ., Burnette, CP., Kadivar, H., Stone, WL., Wallace, MT. (2010) An extended multisensory temporal binding window in autism spectrum disorders. Experimental Brain Research, 203 (2) 381-9.

Dean, P., Redgrave, P., Westby, GW. (1989) Event or emergency? Two response systems in the mammalian superior colliculus. Trends in Neuroscience,12 (4)13747.

Dickinson, A., Jones, M., Milne, E. (2014). Oblique orientation discrimination thresholds are superior in those with a high level of autistic traits. Journal of Autism and Developmental Disorders, 44 (11) 2844-2850.

Donohue, SE., Woldor MG., Mitro, SR. (2010) Video game players show more precise 
multisensory temporal processing abilities. Attention, Perception, \& Psychophysics, 72 (4) 1120-1129.

Donohue, SE. Darling, EF., Mitro, SR. (2012) Links between multisensory processing and autism. Experimental Brain Research 222 (4) 377-387.

Falter, CM., Elliott, MA., Bailey, AJ. (2012) Enhanced visual temporal resolution in autism spectrum disorders. PLoS One 7 (3) e 32774.

Faraone, SV., Biederman, J. (2005) What is the prevalence of adult ADHD? Results of a population screen of 966 adults. Journal of Attention Disorders, 9 (2) 384-391.

Faraone, SV., Sergeant, J., Gillberg, C., Biederman, J. (2003) The worldwide prevalence of ADHD: is it an American condition? World Psychiatry, 2 (2) 104113.

Fink, MP., Ulbrich, J., Churan, Wittmann, M. (2006) Stimulus-dependent processing of temporal order. Behavioural Processes, 71 (2) 344-352.

Ford, T., Goodman, R., Meltzer, H. (2003) The British Child and Adolescent Mental Health Survey 1999: the prevalence of DSM-IV disorders. Journal of the American Academy of Child and Adolescent Psychiatry 42 (10) 1203-1211.

Frassinetti, F., Bolognini, N., Làdavas, E. (2002) Enhancement of visual perception by crossmodal visuo-auditory interaction. Experimental Brain Research, 147 (3) 332343.

Fujisaki, W., Shimojo, S., Kashino, M., Nishida, S. (2004) Recalibration of audiovisual simultaneity. Nature Neuroscience, 7 (7) 773-778.

Garca-Perez, MA., Alcala-Quintana, R. (2012) On the discrepant results in synchrony judgment and temporal-order judgment tasks: a quantitative model. Psychonomic Bulletin \& Review, 19 (5) 820-846.

Hairston, WD., Burdette, JH., Flowers, DL., Wood, FB., Wallace, MT. (2005) Altered 
temporal profile of visual-auditory multisensory interactions in dyslexia, Experimental Brain Research, 166 (3-4) 474-480.

Hassan, DM. Azzam, H. (2012) Sensory integration in attention deficit hyperactivity disorder: Implications to postural control. In: Contemporary Trends in ADHD Research, Norvilitis, JM. (ed). In Tech (online), 1-12.

Hudziak, JJ., Achenbach, TM., Althoff, RR., Pine, DS. (2007) A dimensional approach to developmental psychopathology. International Journal of Methods in Psychiatric Research, 16 (S1), S16-S23.

Kessler, RC., Adler, L., Ames, M., Demler, O., Faraone, S., Hiripi, E., Howes, MJ., Jin, R., Secnik, K., Spencer, T., Ustun, TB., Walters, EE. (2005) The World Health Organization Adult ADHD Self-Report Scale (ASRS): a short screening scale for use in the general population. Psychological Medicine, 35 (2) 245-56.

Koelewijn, TA., Bronkhorst, J., Theeuwes (2010) Attention and the multiple stages of multisensory integration: A review of audiovisual studies. Acta Psychologica, 134 (3) $372-384$.

Koziol, LF. D. Budding, D. (2012) ADHD and sensory processing disorders: placing the diagnostic issues in context. Applied Neuropsychology: Child 1, (2) 137-144.

Laasonen, J., Tomma-Halme, P., Lahti-Nuuttila, E., Service, V. Virsu (2000) Rate of information segregation in developmentally dyslexic children, Brain and Language, 75 (1) 66-81.

Larsson, H., Anckarsater, H., Råstam, M., Chang, Z., Lichtenstein, P. (2012). Childhood attention-deficit hyperactivity disorder as an extreme of a continuous trait: a quantitative genetic study of 8,500 twin pairs. Journal of Child Psychology and Psychiatry, 53, 73-80.

Levy, F., Hay, DA., McStephen, M., Wood, C., Waldman, I. (1997). Attention-Deficit 
hyperactivity disorder: A category or a continuum? genetic analysis of a LargeScale twin study. Journal of the American Academy of Child \& Adolescent Psychiatry, 36, 737-744.

Love, SA. Petrini, K., Cheng, A., Pollick, FE. (2013) A psychophysical investigation of differences between synchrony and temporal order judgments. PLoS One 8, (1) e54798.

Martin, J., Hamshere, ML., Stergiakouli, E., O'Donovan, M. C., Thapar, A. (2014). Genetic risk for attention-deficit/hyperactivity disorder contributes to neurodevelopmental traits in the general population. Biological Psychiatry, 76, 664-671.

Marusich, LR., Gilden, DL. (2014) Assessing temporal integration spans in ADHD through apparent motion. Neuropsychology, 28 (4) 585-93.

Mathôt, S., Schreij, D., Theeuwes, J. (2012) OpenSesame: an open-source, graphical experiment builder for the social sciences. Behavior Research Methods 44 (2) 314324.

Megevand, P., Molholm, S., Nayak, A., Foxe, JJ. (2013) Recalibration of the multisensory temporal window of integration results from changing task demands. PLoS One 8 (8) e71608.

inten (1987) Determinants of multisensory integration in superior colliculus neurons. I. Temporal factors. Journal of Neuroscience, 7 (10) 3215-3229.

Michalek, AM., Watson, SM., Ash, I., Ringleb, S., Raymer, A. (2014) Effects of noise and audiovisual cues on speech processing in adults with and without ADHD. International Journal of Audiology, 53 (3) 145-152.

Mongillo, EA., Irwin, JR., Whalen, D., Klaiman, C., Carter, AS., Schultz, RT. (2008) Audiovisual processing in children with and without autism spectrum disorders, Journal of Autism and Developmental Disorders, 38 (7) 1349-1358. 
Miller, LM., D'Esposito, M. (2005) Perceptual fusion and stimulus coincidence in the cross-modal integration of speech. Journal of Neuroscience, 25 (25) 5884-93.

Noesselt, T., Rieger, JW., Schoenfeld, MA., Kanowski, M., Hinrichs, H., Heinze, HJ., Driver, J. (2007) Audiovisual temporal correspondence modulates human multisensory superior temporal sulcus plus primary sensory cortices. Journal of Neuroscience, 27 (42) 11431-41.

Overton, P. G. (2008) Collicular dysfunction in attention deficit hyperactivity disorder. Medical Hypotheses, 70, 1121-1127.

Overton, P.G., Clements, KM. (2009) Does collicular dysfunction underlie distractibility in Attention Deficit Hyperactivity Disorder? In: Advances in psychology research, Columbus AM. (ed) Nova Science Publishers: New York, 193-210.

Petrini, K., Dahl, S., Rocchesso, D., Waadeland, CH., Avanzini, F., Puce, A., Pollick, FE. (2009) Multisensory integration of drumming actions: musical expertise affects perceived audiovisual asynchrony. Experimental Brain Research, 198 (2-3) $339-52$.

Polanczyk, GM., de Lima, SS., Horta, BLL. Biederman, J., Rohde, LAA. (2007) The worldwide prevalence of ADHD: a systematic review and metaregression analysis. American Journal of Psychiatry, 164 (6) 942-948.

Polner, B., Aichert, D., Macare, C., Costa, A., Ettinger, U. (2015). Gently restless: association of ADHD-like traits with response inhibition and interference control. European Archives of Psychiatry and Clinical Neuroscience, 265 (8) 689-99.

Powers, AR., Hillock, AR., Wallace, MT. (2009) Perceptual training narrows the temporal window of multisensory binding, Journal of Neuroscience, 29 (39) 12265-12274. 
Powers, AR. Hevey, MA., Wallace, MT. (2012) Neural correlates of multisensory perceptual learning, Journal of Neuroscience 32 (18) 6263-6274.

Reuter, M., Kirsch, P., Hennig, J. (2006) Inferring candidate genes for attention deficit hyperactivity disorder (ADHD) assessed by the World Health Organization Adult ADHD Self-Report Scale (ASRS). Journal of Neural Transmission, 113 (7) 92938.

Rodriguez, A., Jarvelin, MR., Obel, C., Taanila, A., Miettunen, J., Moilanen, I.. . . Olsen, J. (2007) Do inattention and hyperactivity symptoms equal scholastic impairment? evidence from three European cohorts. BMC Public Health, 7 (1) $327-9$.

Rubenstein, J., Merzenich, M. (2003) Model of autism: increased ratio of excitation/ inhibition in key neural systems. Genes, Brain and Behavior, 2 (5) 255-267.

Schneider, KA., Bavelier, D. (2003) Components of visual prior entry. Cognitive Psychology, 47 (4) 333-366.

Seixas, M., Weiss, M., Müller, U. (2012) Systematic review of national and international guidelines on attention deficit hyperactivity disorder. Journal of Psychopharmacology, 26 (6) 753-765.

Shore, DI., Spence, C., Klein, RM. (2001) Visual prior entry. Psychological Science 12 (3) $205-212$.

Smith, WF. (1933) The relative quickness of visual and auditory perception. Journal of Experimental Psychology, 16 (2) 239-257.

Smith, A., Taylor, E., Warner Rogers, J., Newman, S., Rubia, K. (2002) Evidence for a pure time perception deficit in children with ADHD. Journal of Child Psychology and Psychiatry, 43 (4) 529-542.

Spence, C., Shore, DI., Klein, RM. (2001) Multisensory prior entry. Journal of 
Experimental Psychology: General 130 (4) 799-832.

Spencer, TJ., Biederman, J., Wilens, TE., Faraone, SV. (2002) Overview and neurobiology of attentiondeficit/hyperactivity disorder. Journal of Clinical Psychiatry, 63 (Suppl 12) 3-9.

Stark R., Bauer E., Merz CJ., Zimmermann M., Reuter M., Plichta MM., Kirsch P., Lesch KP., Fallgatter AJ., Vaitl D., Herrmann MJ. (2011) ADHD related behaviors are associated with brain activation in the reward system. Neuropsychologia, 49 (3) 426-34.

Stevenson, RA., Altieri, NA., Kim, S., Pisoni, DB., James, TW. (2010) Neural processing of asynchronous audiovisual speech perception. Neuroimage, 49 (4) 3308-18.

Stevenson, RA., Wilson, MM., Powers, AR., Wallace, MT. (2013) The effects of visual training on multisensory temporal processing. Experimental Brain Research, 225 (4) $479-489$.

Stevenson, RA., Zemtsov, RK., Wallace, MT. (2012) Individual differences in the multisensory temporal binding window predict susceptibility to audiovisual illusions. Journal of Experimental Psychology. Human Perception and Performance, 38 (6) 1517-1529.

Stone, J., Hunkin, N., Porrill, J., Wood, R., Keeler, V., Beanland, M., Port, M., Porter, N. (2001) When is now? perception of simultaneity. Proceedings of the Royal Society of London. Series B: Biological Sciences, 268 (1462) 31-38.

Tannock, R. (1998) Attention deficit hyperactivity disorder: advances in cognitive, neurobiological, and genetic research. Journal of Child Psychology and Psychiatry, and Allied Disciplines, 39 (1) 65-99.

Thorley, G. (1984) Hyperkinetic syndrome of childhood - clinical characteristics. 
British Journal of Psychiatry, 144, 16-24.

Toplak, ME., Dockstader, C., Tannock, R. (2006) Temporal information processing in adhd: findings to date and new methods. Journal of Neuroscience Methods, 151 (1) $15-29$.

Valko, L. Schneider, G., Doehnert, M., Müller, U., D. Brandeis, Steinhausen, H.-C., Drechsler, R. (2010) Time processing in children and adults with ADHD. Journal of Neural Transmission, 117 (10) 1213-1228.

van Eijk, RL., Kohlrausch, A., Juola, JF., van de Par, S. (2008) Audiovisual synchrony and temporal order judgments: effects of experimental method and stimulus type, Perception \& Psychophysics, 70 (6) 955-968.

Vatakis, A. Navarra, J., Soto-Faraco, S., Spence, C. (2008) Audiovisual temporal adaptation of speech: temporal order versus simultaneity judgments. Experimental Brain Research, 185 (3) 521-529.

Vatakis, A. Navarra, S., Soto-Faraco, S., Spence, C. (2007) Temporal recalibration during asynchronous audiovisual speech perception, Experimental Brain Research, $181(1) 173-181$.

Wittmann, M., Simmons, AN., Flagan, T., Lane, SD., Wackermann, J., Paulus, MP. (2011) Neural substrates of time perception and impulsivity, Brain Research, 1406 43-58.

Yarrow, K., Shapiro, S., DiCosta, S., Arnold, DH. (2014) A 2AFC simultaneityjudgment task to estimate the point of subjective simultaneity. Procedia - Social and Behavioral Sciences $126(0)$ 66, International Conference on Timing and Time Perception, 31 March 3 April 2014, Corfu, Greece.

Yochman, A., Parush, S., Ornoy, A. (2004) Responses of preschool children with and without adhd to sensory events in daily life. American Journal of Occupational 
Therapy, 58 (3) (2004) 294-302.

Zampini, M., Shore, DI., Spence, C. (2003) Audiovisual temporal order judgments, Experimental Brain Research, 152 (2) 198-210.

Zampini, M., Guest, S., Shore, DI, Spence, C. (2005) Audio-visual simultaneity judgments. Perception \& Psychophysics, 67 (3) 531-544. 


\section{Abstract}

Abnormalities in multimodal processing have been found in many developmental disorders such as autism and dyslexia. However, surprisingly little empirical work has been conducted to test the integrity of multisensory integration in Attention Deficit Hyperactivity Disorder (ADHD). The main aim of the present study was to examine links between symptoms of ADHD (as measured using a self-report scale in a healthy adult population) and the temporal aspects of multisensory processing. More specifically, a Simultaneity Judgment (SJ) and a Temporal Order Judgment (TOJ) task were used in participants with low and high levels of ADHD-like traits to measure the temporal integration window and Just-Noticeable Difference (JND) (respectively) between the timing of an auditory beep and a visual pattern presented over a broad range of stimulus onset asynchronies. The Point of Subjective Similarity (PSS) was also measured in both cases. In the SJ task, participants with high levels of ADHD-like traits considered significantly fewer stimuli to be simultaneous than participants with high levels of ADHD-like traits, and the former were found to have significantly smaller temporal windows of integration (although no difference was found in the PSS in the SJ or TOJ tasks, or the JND in the latter). This is the first study to identify an abnormal temporal integration window in individuals with ADHD-like traits. Perceived temporal misalignment of two or more modalities can lead to distractibility (e.g., when the stimulus components from different modalities occur separated by too large of a temporal gap). Hence, an abnormality in the perception of simultaneity could lead to the increased distractibility seen in ADHD. 
Evidence suggest that the superior colliculus (SC) is dysfunctional in ADHD

Multisensory integration - involving the SC - has not been studied empirically in ADHD before

We report a narrowing of the temporal window of sensory integration in subclinical ADHD

Distractibility in ADHD may arise in part from a failure to integrate multimodal stimuli 